\section{Health status and quality of life in patients with severe hemophilia A: A cross-sectional survey}

\author{
Majid Davari, ${ }^{1,2}$ Zahra Gharibnaseri, ${ }^{3}$ \\ Roya Ravanbod, ${ }^{4}$ Abolfazl Sadeghi ${ }^{1}$ \\ ${ }^{1}$ Department of Pharmacoeconomics \\ and Pharmaceutical Administration, \\ Faculty of Pharmacy, Tehran University \\ of Medical Sciences, Tehran; ${ }^{2}$ Health \\ Equity Research Center, Tehran \\ University of Medical Science, Tehran; \\ ${ }^{3}$ Department of Health Economics, \\ National Institute for Health Research, \\ Tehran University of Medical Science, \\ Tehran; ${ }^{4}$ Department of Physiotherapy, \\ Faculty of Medical Sciences, Tarbiat \\ Modares University, Tehran, Iran
}

\begin{abstract}
Among different groups of hemophiliacs, those suffering from Severe Hemophilia A (SHA) are most vulnerable to the complications of the disease. This study investigated the Health-Related Quality of Life (HR-QoL) among adult patients with SHA. A cross-sectional study was designed to gather demographic and clinical information from adult patients with SHA. Patients with inhibitors were excluded. The remaining were asked to complete the HR-QoL questionnaire after being examined for joint health using the Hemophilia Joint Health Score (HJHS). The HR-QoL and joint conditions were measured in 38 patients. The mean EQ-5D value scores were 0.46 $(\mathrm{SD}=0.23)$ while the mean Visual Analogous Scale score was $50(\mathrm{SD}=18.7)$. The clinical examination of patients indicated that the HJHS were as follows: eight patients had a score of $55-75,12$ patients had a score of 40-55, 7 of them (25-40) and 11 patients had a score of 10-25. The results obtained from this study showed that HRQoL in hemophilia patients was considerably low. Pain, anxiety/depression, and motion limitations were the main causes of the disutility for these patients respectively.
\end{abstract}

\section{Introduction}

Hemophilia A (HA) is the most common severe form of congenital coagulopathies and is characterized by a deficiency of factor eight (FVIII), which has serious consequences for the activation of the endogenous system of the coagulation cascade. $^{1}$

The prevalence of hemophilia A (per 100,000 males) varies from 0.05 in Nigeria to 38.6 in Iceland. ${ }^{2}$ Hemophilia A and B in Iran with an estimated prevalence rate of 14 and 2.5 in 100,000 males respectively are listed in the rare disease category. ${ }^{3}$

Based on the activity level of FVIII, the disease severity of HA is classified as severe: $<1 \%$ of the normal FVIII activity, moderate: $1-5 \%$ of the normal FIX activity, and mild: $>5 \%$ and $<40 \%$ of the normal factors. ${ }^{4}$ The national registry database of the Iranian Ministry of Health has recorded 4700 hemophilia patients in Iran with FVIII deficiency $(82 \%)$ of which $60 \%$ are categorized as SHA. ${ }^{5}$ Based on the data provided, it can be understood that SHA comprises the largest group in the total population of hemophilia patients. It should also be noted that due to spontaneous or traumatic hemorrhages that happen in $80 \%$ of the joints, ${ }^{6}$ these patients are most vulnerable to the complications of the disease. Prior to the 1960s, when the blood transfusion was the only treatment available to hemophilia patients, mortality and morbidity rates caused a great burden of disease, particularly in the severe subgroup with a life expectancy of 11 years. However, improved access to factor concentrates has increased the life expectancy of severe hemophiliacs to 56.8 years. $^{7-9}$ Consequent upon the chronic nature of the disease and the complications, particularly arthritis, causing physical impairment, this disease has serious impact on patients' Quality of Life (QoL). Measuring the QoL makes it possible to evaluate patients' overall well-being. This study aimed to investigate the HealthRelated quality of life (HR-QoL) among adult patients with SHA, registered in Tehran clinics.

\section{Materials and Methods}

In order to gather demographic and clinical information from patients with severe HA, a cross-sectional study was designed.

\section{Data source}

We gathered data from adult patients registered with SHA in Tehran hemophilia clinics. These centers include the Iranian comprehensive hemophilia care center (ICHCC) and the comprehensive hemophilia care center in Imam Khomeini Hospital. Patients were contacted via their registered phone number. Patients willing to participate in the study, were invited to the physiotherapy ward at the ICHCC for face-to-
Correspondence: Abolfazl Sadeghi, Department of Pharmacoeconomics and Pharmaceutical Administration, Faculty of Pharmacy, Tehran University of Medical Sciences, Tehran, Iran.

Tel: +98.21.88896696.

E-mail: abolfazlsadeghi32@gmail.com

Key words: Hemophilia, health-related quality of life, hemophilia joint health score, EQ-5D.

Contributions: MD designed the study; RR and $\mathrm{ZG}$ performed the measurements, AS wrote the paper with input from all authors.

Conflict of interest: the authors declare no potential conflict of interest.

Funding: none.

Received for publication: 29 September 2018 Accepted for publication: 15 February 2019.

This work is licensed under a Creative Commons Attribution-NonCommercial 4.0 International License (CC BY-NC 4.0).

(C) Copyright M. Davari et al., 2019

Licensee PAGEPress, Italy

Hematology Reports 2019; 11:7894

doi:10.4081/hr:2019.7894

face interview as well as clinical examination. In order to estimate the Health Related Quality of Life (HR-QoL) patients were asked to complete QoL questionnaires. Our physiotherapist conducted a $30 \mathrm{~min}$ the examination of patients' joints.

\section{Patients' characteristics}

Hemophilia A patients, 18 years and older, with FVIII levels less than 0.01 IU.ml ${ }^{-1}$ were included in the study leaving out moderate or mild severity cases. Any patient with a history of inhibitors (any inhibitor activity $>0.6$ Bethesda Units) was excluded.

\section{Activity level}

Patients' job types and their usual activity level was obtained via a modified Tegner activity level scale. This scoring system is composed of a list of daily living activities and sports, ranging from zero presenting "sick leave or disability pension because of knee problems," to a score of ten corresponding to participation in national and international elite competitive sports. The multiple levels of activity were read and explained to the patient and patients were asked to select the level that best describes their current level of activity.

\section{Joint score}

The Hemophilia Joint Health Score 
(HJHS 2.1) was chosen based on its comprehensive feature. HJHS correlates appropriately with bleeding rates $(\mathrm{rs}=0.50)$ and physician global assessment of joint health ( $\mathrm{rs}=0.42$ ) as well as radiographic changes. The scoring was performed by a trained physiotherapist. The physiotherapist examined the patients' six hinged joints and reported the overall joint health state with a number between 0 and 124 , representing full health and all joints deteriorated respectively.

\section{Quality of life}

A validated version of the Persian translated EQ-5D-3L questionnaire was used, since it has shown construct validity and responsiveness to change. ${ }^{10} \mathrm{EQ}-5 \mathrm{D}-3 \mathrm{~L}$ is a questionnaire designed to give a descriptive system of the health-related quality of life regarding five dimensions. These include the following dimensions; mobility, selfcare, usual activities, pain/discomfort, and anxiety/depression. Furthermore; each dimension is presented at three levels: no problems, some problems, extreme problems. As a result of the absence of locally appropriate setup values, the European EQ$5 \mathrm{D}$ value set was used in order to measure the patient's quality of life.

\section{Results}

After making an inquiry about the registry centers' list for male patients with FVIII deficiency who had visited the centers recently, 186 patients were identified within the age range of 18-57 years old. From this total, 92 were inaccessible through telephone calls, 22 patients were diagnosed with mild or moderate severity and 16 patients had a history of inhibitors (any inhibitor activity $>0.6$ Bethesda Units), all of which were excluded from the study.

The 56 remaining patients were invited for a face-to-face interview at the ICHCC center. Of this number, 15 patients did not show up on the interview days and three patients responded incompletely leaving 38 reports for analysis. The distribution of patients by different parameters is presented in Table 1 .

\section{Quality of life index}

The quality of life using the Visual Analogue Scale (VAS) indicated an average score of $50(\mathrm{SD}=18.7)$.

Using the European value set, the HRQoL index ranged between 0.08 and 0.8 showing a large variety among the patients. More details on the utility index is present-

Table 1. Patients characteristics.

\begin{tabular}{lcc} 
Parameter & Category & Number (\%) \\
Age (years) & $18-27$ & $8(20)$ \\
& $28-37$ & $16(39)$ \\
& $38-47$ & $8(20)$ \\
BMI $\left(\mathrm{kg} / \mathrm{m}^{2}\right)$ & $48-57$ & $9(22)$ \\
& $<18.5$ & $8(20)$ \\
& $18.5-24.9$ & $17(41)$ \\
\hline Activity level & $>25$ & $16(39)$ \\
& Light work & $18(44)$ \\
& Moderate & $11(27)$ \\
& Heavy work & $12(29)$
\end{tabular}

Table 2. EQ-5D Index and Visual Analogue Scale (VAS) Scores.

\begin{tabular}{|c|c|c|c|c|c|c|}
\hline \multirow{2}{*}{ Descriptive Statistics } & \multirow[t]{2}{*}{ N. } & \multirow[t]{2}{*}{ Min } & \multirow[t]{2}{*}{ Max } & \multicolumn{2}{|c|}{ Mean } & \multirow[t]{2}{*}{ Std. Deviation } \\
\hline & & & & Statistic & Std. Error & \\
\hline VAS Score & 37 & 10.00 & 90.00 & 50.3378 & 3.07892 & 18.72834 \\
\hline Utility & 37 & 0.0827 & 0.8062 & 0.458335 & 0.0387694 & 0.2358252 \\
\hline Valid N (list wise) & 36 & & & & & \\
\hline
\end{tabular}

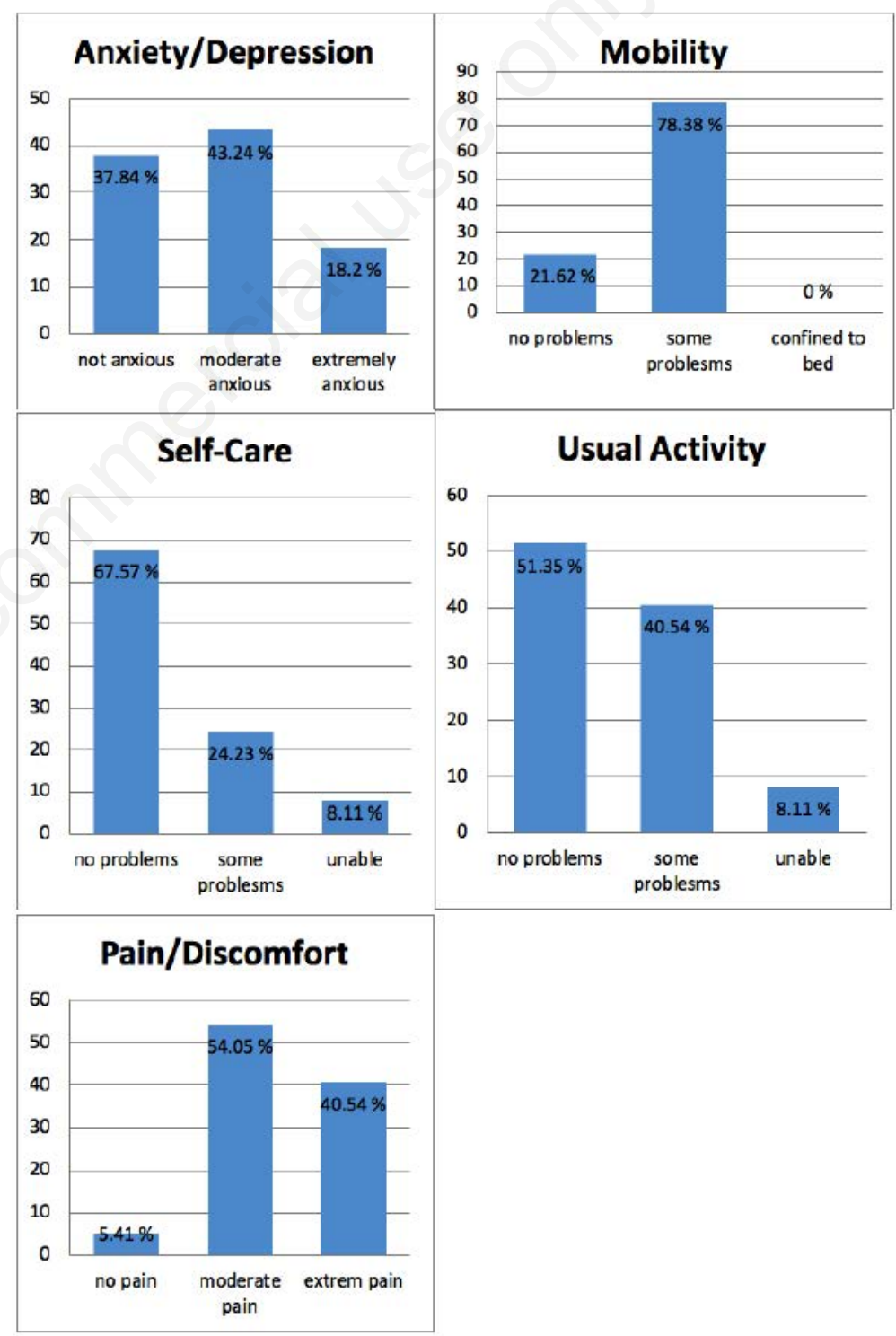

Figure 1. EQ-5D-3L health domains. 
ed in Table 2 and Figure 1.

\section{Hemophilia joint health score}

The clinical examination of patients indicated that the HJHS scores are as follows: eight patients had a score of 55-75, 12 patients had a score of $40-55,7$ of the $25-40$ and 11 patients had a score of 10-25. Figure 2 shows the average joint score of patients' six hinged joints.

\section{Discussion}

Prior to the 60 s, when the blood transfusion was the only treatment available for hemophilia patients, mortality and morbidity rates resulting in a great burden of disease, particularly in the severe subgroup with a life expectancy of 11 years. However, improved access to factor concentrates has increased the life expectancy of severe hemophiliacs to 56.8 years..$^{7-9}$

The quality of life in hemophilia patients requires thorough attention, due to the chronic nature of the disease. Measuring the QoL gives us the possibility of assessing patients' treatment process. We aimed at investigating the QoL among adult hemophilia patients registered in Tehran clinics.

Our study revealed that the average utility score for patients is 0.46 standing at a lower level than the general population. This result is similar to previously published studies reporting QoL in hemophilia patients. ${ }^{11,12}$

Faranoush et al. showed that over 50\% of adult patients with severe hemophilia A in Tehran, report a low to moderate level of QoL. ${ }^{13}$ The questionnaire used in this study is the A36 Hemofilia-QoL, which is a disease-specific tool for quantifying HR-QoL. The disease-specific questionnaires are more effective compared to generic questionnaires in detecting subtle changes in HR-QoL. ${ }^{14}$ However, generic questionnaires are necessary in order to compare the state of QoL in patients and the general population or patients suffering from different diseases. Our study indicated that $95 \%$ of the patients reported moderate to severe pain and discomfort, making the HR-QoL level most negatively affected by this domain.

Patients who chose the moderate/severe level of anxiety/depression were less than those who chose similar levels in mobility. However, according to the value set used in this research, the negative coefficient of anxiety/depression on level three is higher than that of mobility. Therefore, anxiety/depression has turned out as the second negative factor affecting hemophilia patients' HR- QoL.

Our clinical examinations using the HJHS questionnaire showed that the right and left knees with joint scores of 8.6 and 7.06 units, respectively, had the worst situation among all hinged joints. According to Poon et al.'s study, motion limitations due to joint conditions in hemophilia patients caused a decrease in QoL scores. ${ }^{15}$ While the present study has supplied useful information about the HR-QoL of hemophilia patients, it has few limitations that must be considered. The first is the small patient population which is due to the rare nature of the disease and the minority of patients willing to take part in the study. In order to minimize the effects of this limitation, stratified sampling was used in an attempt to reflect the age distribution in the setting of the study that was conducted. The collection of samples from all hemophilia centers located in Tehran was also considered. Another limitation is that this study did not measure the impact of age on hemophiliacs

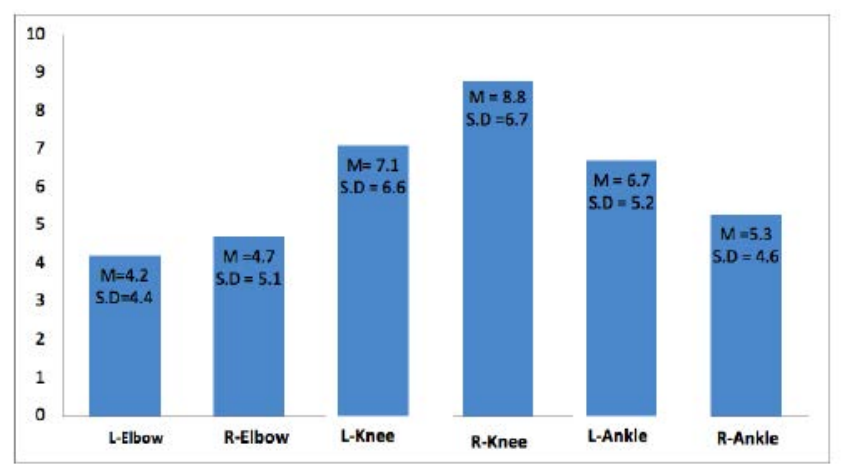

Figure 2. Average joint score of patients' hinged joints. L: Left, R: Right.

QoL; which has been shown by Forsyth et al. that patients report higher disability and pain at increased age, resulting in lower HR-QoL. ${ }^{16}$ In addition, Trippoli et al. have shown that age significantly influenced both the EuroQoL and the SF-36 scores. ${ }^{17}$

\section{Conclusions}

The results obtained from this study showed that HR-QoL of the hemophilia patients was considerably low. Pain, anxiety/depression, and motion limitations were the main causes of the disutility for these patients respectively. These findings suggest that providing appropriate psychotherapy, pain management services, and physiotherapy services, could improve HR-QoL of the hemophilia patients significantly.

\section{References}

1. Li T, Miller $\mathrm{CH}$, Driggers J, et al. Mutation analysis of a cohort of US patients with hemophilia B. Am j hematol 2014,89:375-9.

2. Stonebraker JS, Bolton-Maggs PH, Michael Soucie J, et al. A study of variations in the reported haemophilia A prevalence around the world. Haemophilia 2010;16:20-32.

3. Mehdizadeh M, Kardoost M, Zamani $\mathrm{G}$, et al. Occurrence of haemophilia in Iran. Haemophilia 2009;15:348-51.

4. White GC, Rosendaal F, Aledort LM, et al. Definitions in hemophilia. Recommendation of the scientific subcommittee on factor VIII and factor IX of the scientific and standardization committee of the International Society on Thrombosis and Haemostasis. Thromb Haemost 2001;85:560.

5. Gharibnaseri Z, Davari M, Cheraghali A, et al. Health care resource utilization and cost of care for haemophilia A and B patients in Iran. Transfus Apher Sci 2016;54:122-6.

6. Oldenburg J, Hertfelder HJ. [Blood coagulation and hemorrhagic diathesis] Pharm Unserer Zeit 2006;35:20-8.

7. Larsson SA. Life expectancy of Swedish haemophiliacs, 1831-1980. Br J Haematol 1985;59:593-602.

8. Darby SC, Ewart DW, Giangrande PL, Spooner RJ, Rizza CR, Dusheiko GM, et al. Mortality from liver cancer and liver disease in haemophilic men and boys in UK given blood products contaminated with hepatitis $\mathrm{C}$. The Lancet 1997;350:1425-31.

9. Word Health Organization. World health statistics. In. Geneva; WHO: 2013. 
10. Brazier J, Roberts J, Tsuchiya A, Busschbach J. A comparison of the $\mathrm{EQ} \square 5 \mathrm{D}$ and $\mathrm{SF} \square 6 \mathrm{D}$ across seven patient groups. Health Econ 2004,13:873-84.

11. Varaklioti A, Kontodimopoulos N, Niakas D, et al. Health-Related Quality of Life and Association with Arthropathy in Greek Patients with Hemophilia. Clin Appl Thromb Hemostasis 2017:1076029617733041.

12. Miners AH, Sabin C, Tolley K, et al. Assessing health-related quality-of-life in patients with severe haemophilia A and B. Psychol Health Med 1999;4:515.
13. Faranoush M, Shahverdi E, Ghorbani R, Moghaddam M. Health-related quality of life in Iranian adult men with severe hemophilia. Blood Coagul Fibrinolysis 2017;28:638-41.

14. de Vries M, Ouwendijk R, Kessels AG, et al. Comparison of generic and disease-specific questionnaires for the assessment of quality of life in patients with peripheral arterial disease. J Vasc Surg 2005;41:261-8.

15. Poon Jl, Zhou Zy, Doctor J, et al. Quality of life in haemophilia A: Hemophilia Utilization Group Study Va (HUGS-Va). Haemophilia 2012;18:699-707.
16. Forsyth AL, Witkop M, Lambing A, et al. Associations of quality of life, pain, and self-reported arthritis with age, employment, bleed rate, and utilization of hemophilia treatment center and health care provider services: results in adults with hemophilia in the HERO study. Patient Prefer Adherence 2015;9:1549.

17. Trippoli S, Vaiani M, Linari S, et al. Multivariate analysis of factors influencing quality of life and utility in patients with haemophilia. Haematologica 2001;86:722-8. 